\title{
LOS BOLOS EPIDURALES INTERMITENTES PROGRAMADOS PARA MANTENIMIENTO DE LA ANALGESIA DE PARTO. ¿PROLONGAN LOS TIEMPOS DEL TRABAJO DE PARTO?
}

\section{THEINTERMITTENTEPIDURALBOWLSPROGRAMMEDFORTHEMAINTENANCE OF LABOR ANALGESIA. ARE THE TIMES OF LABOR EXTENDED?}

\author{
Eddisson Quispe Pilco 1,a
}

Estimado Señor editor:

Luego de haber leído con mucho interés el articulo "Bolos epidurales intermitentes programados para mantenimiento de la analgesia del trabajo de parto: estudio observacional, analítico de tipo cohorte" por Calderón Lozano Marjorie et al. publicado en el volumen 9 número 3 de la Revista Peruana de la Investigación Materno Perinatal (INMP), considero de suma importancia poder responder la pregunta si realmente esta técnica de mantenimiento de analgesia prolonga los tiempos del trabajo de parto teniendo en cuenta la información actual.

La analgesia epidural actualmente es la técnica más segura y eficaz con la que contamos para controlar el dolor de parto ${ }^{1}$. Ya se ha comprobado seguridad materna fetal ${ }^{1,2}$ y en general no prolonga los tiempos del trabajo de parto, solo se la asocia con un aumento del parto instrumentado en el expulsivo ${ }^{1}$. El mantenimiento de la analgesia por medio del catéter epidural es hasta hoy un tema de controversia y estudio, se ha comprobado que la técnica de bolos intermitentes produce un mejor bloqueo analgésico y menos complicaciones que la técnica de infusión continua ${ }^{3}$.

La duración del trabajo de parto es influida por múltiples factores como edad, paridad, grado de distensibilidad de tejidos del canal de parto, uso de oxitocina, variedad de presentación fetal, tamaño fetal, ruptura de membranas fetales, intensidad de dolor, capacidad de pujar adecuadamente, entre otras.

El artículo reporta prolongación de los tiempos de la dilatación y el expulsivo con el uso de bolos epidurales intermitentes programados (BEIP), pero probablemente como la autora refiere existiría un sesgo por tener el grupo de analgesia un mayor numero de nulíparas respecto al grupo de control que tiene una mayor proporción de multíparas, ya que las nulíparas se caracterizan por tener tiempos de parto mas prolongados y dolorosos respecto a las multíparas ${ }^{4,5}$.

Tampoco se menciona la variedad de presentación fetal que en caso de ser derechas y/o posteriores son consideradas como distócicas y generalmente solicitan más analgesia porque son más dolorosas y causan tiempos de parto más prolongados debido a la mayor rotación que deben realizar en el canal de parto ${ }^{4}$.

Por tanto se concluye que son necesarios más estudios respecto a la técnica de BEIP y ser muy rigurosos en los criterios de inclusión y exclusión para uniformizar la comparación de muestras. Solo así podremos determinar si la técnica de BEIP realmente prolonga los tiempos del parto respecto a las gestantes que no reciben analgesia epidural.

Declaración de conflictos de intereses: El autor declara no tener conflicto de interés.

Financiamiento: Autofinanciado

\footnotetext{
Instituto Nacional Materno Perinatal, Lima - Perú

a Médico Anestesiólogo.
}

(iD) Código ORCID: https://orcid.org/0000-0002-2854-7329, Eddisson Quispe Pilco

Citar como: Quispe Pillco E. Los bolos epidurales intermitentes programados para mantenimiento de la analgesia de parto ¿Prolongan los tiempos del trabajo de parto?. Rev Peru Investig Matern Perinat. 2021; 10(3): 9 -10 DOI https://doi.org/10.33421/inmp.2021237 


\section{REFERENCIAS BIBLIOGRÁFICAS}

1. Ashagrie $\mathrm{H}$, Fentie $\mathrm{D}$, Kassahun $\mathrm{H}$. A review article on epidural analgesia for labor pain management: A systematic review. International Journal of Surgery Open 2020; 24:100104. https://doi.org/10.1016/j.ijso.2020.04.007.

2. Anim-Somuah M, Smyth RMD, Cyna AM, Cuthbert A.

Epidural versus non-epidural or no analgesia for pain management in labour. Cochrane Database of Systematic Reviews 2018, Issue 5. Art. No.: CD000331. DOI: 10.1002/14651858.CD000331.pub4.

3. Nanji J, Carvalho B. Pain management during labor and vaginal birth. Best Practice \& Research Clinical Obstetrics and Gynaecology 2020; 67:100-112. https://doi.org/10.1016/j. bpobgyn.2020.03.002.

4. Lim G, Facco F, Nathan N, Waters J, Wong C, Eltzschig H. A Review of the Impact of Obstetric Anesthesia on Maternal and Neonatal Outcomes. Anesthesiology 2018; 129:192215 .

5. Lam K, Leung M, Irwin M. Labour analgesia: update and literature review. Hong Kong Medical Journal 2020; 26:41320. https://doi.org/10.12809/hkmj208632.

6. Carvalho B, George R, Cobb B, McKenzie C, Ripley E. Implementation of Programmed Intermittent Epidural Bolus for the Maintenance of Labor Analgesia. Anesthesia \& Analgesia 2016; 123(4):965-971.

\section{Correspondencia}

Eddisson R. Quispe Pilco

Dirección: Av. General Santa Cruz No 459,

dpto. 1004- Jesús Maria, Lima-Perú

Correo: eddisson.quispe@gmail.com

Teléfono: 991889251 